\title{
Ventajas del uso de la Tecnología en el aula de Clases
}

Miguel Días

Escuela Superior de Educación de Torres Nuevas Escuela Superior de Educación Almeida Garrett - Lisboa migdias@gmail.com Enoc Jessé Castro García-Traductor*

Son varios los trabajos de investigación que reconocen las ventajas en la integración de las tecnologías en la Educación (Papert, 1987; OCDE, 2001 a; UNESCO, 2004; Paiva, 2002; Amante, 2007; Miranda, 2007).

De cualquier manera, creemos que antes de conocer esas ventajas y contextos de utilización, es interesante aceptar el desafío propuesto por Cabero (2006) y reflejarlo en dos dimensiones:

1. En primer lugar conocer las variables previas a contemplar antes de la integración de las tecnologías en la enseñanza;

2. En segundo lugar conocer los aspectos a considerar en la toma de decisiones sobre el uso de las tecnologías en los procesos de enseñanza y aprendizaje.

Conforme podemos ver en la Figura 1, Cabero (2006) identifica cinco tipos de variables que influyen en la posibilidad o no de lograr integrar las tecnologías en la enseñanza:

- Variables evolutivas: relacionadas con la capacidad evolutiva de los utilizadores en el acompañamiento de los desarrollos

\footnotetext{
* Profesor de Matemáticas con Orientación en Física en el Grado de Licenciatura. Honduras, C.A. belza34@hotmail.com
} 
tecnológicos llevando, por ejemplo, a que se asuma la responsabilidad de formación continua;

- Variables fisiológicas: relacionadas con la preocupación de planificación teniendo en cuenta las posibles limitaciones que los utilizadores pueden tener en el momento de interacción con la tecnología.

- Variables culturales: referente a la planificación que debe contemplar una preocupación con valores culturales en el uso de tecnologías, por ejemplo, en procesos de comunicación que, en cualquier momento, amplían el espacio cultural;

- Variables relacionadas con el desarrollo económico: no se trata de comprometer la integración de la tecnología en países menos desarrollados, sino de respetar el punto de partida para que la tecnología encuentre un ambiente perfecto y pueda ser recibida con naturalidad;

- Variables relacionadas con la situación de los sistemas educativos: se inscriben aquí aspectos relacionados con la formación, como por ejemplo, la localización de centros de formación y, sobretodo, las competencias pedagógicas que favorecen los procesos de enseñanza y aprendizaje. En este tipo de variables esta también incluido el tipo de infraestructuras escolares: edificios, electricidad, disponibilidad de red, etc.

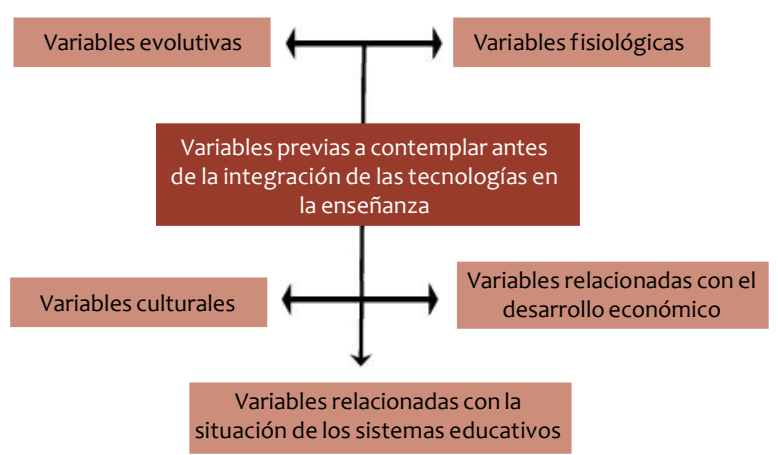

Figura 1- Variables previas a contemplar antes de la integración de las tecnologías en la enseñanza. 
Involucrar tecnologías en la educación hace necesario considerar algunos aspectos singulares relacionados con sus características, sus particularidades comunicativas y sus posibilidades didácticas (Cabero, 2006). La figura 2 representa algunos de esos aspectos:

- El alumno que puede asumir funciones de diseñador, planificador y responsable de su aprendizaje dejando al profesor únicamente como tutor, creador de materiales y evaluador;

- El profesor, como tutor, debe tener competencias no directivas de comunicación de forma a dar la autonomía adecuada al alumno. En este contexto, el papel de tutor consiste en estar disponible para acompañar y ayudar al alumno. El profesor deberá aun poseer competencias sociales para la colaboración para crear materiales, investigación y cambio de información con colegas para enriquecer sus formación;

- Las herramientas son el elemento fundamental en la inserción de las tecnologías en la enseñanza porque por ellas el utilizador interactúa con los sistemas. De entre varios aspectos podemos destacar la disponibilidad creciente de herramientas como software libre que permiten desarrollar varias actividades;

- La metodología a usar en la integración de la tecnología deberá tener en cuenta las características de los recursos tecnológicos de modo que se pueda enriquecer el proceso de enseñanza y aprendizaje. Por eso se deben utilizar metodologías que promuevan la interacción, el trabajo colaborativo y la diversidad de cultura;

- Los equipos evolucionan con gran velocidad y pueden quedar obsoletos en cualquier instante. Por eso deben ser seleccionadas actividades que no exijan la tecnología más reciente.

- A otro nivel, por ejemplo, se debe asegura el acceso rápido a redes para que las actividades no queden comprometidas $y$, en algunos casos, crear alguna resistencia en el uso futuro 
de medios tecnológicos;

- Las posibles funciones a desempeñar por las tecnologías pueden condicionar la decisión sobre el tipo de equipo y tarea;

- Del mismo modo el tipo de comunicación que se pretende utilizar, síncrona o asíncrona, está sujeto a la disponibilidad de recursos tecnológicos.

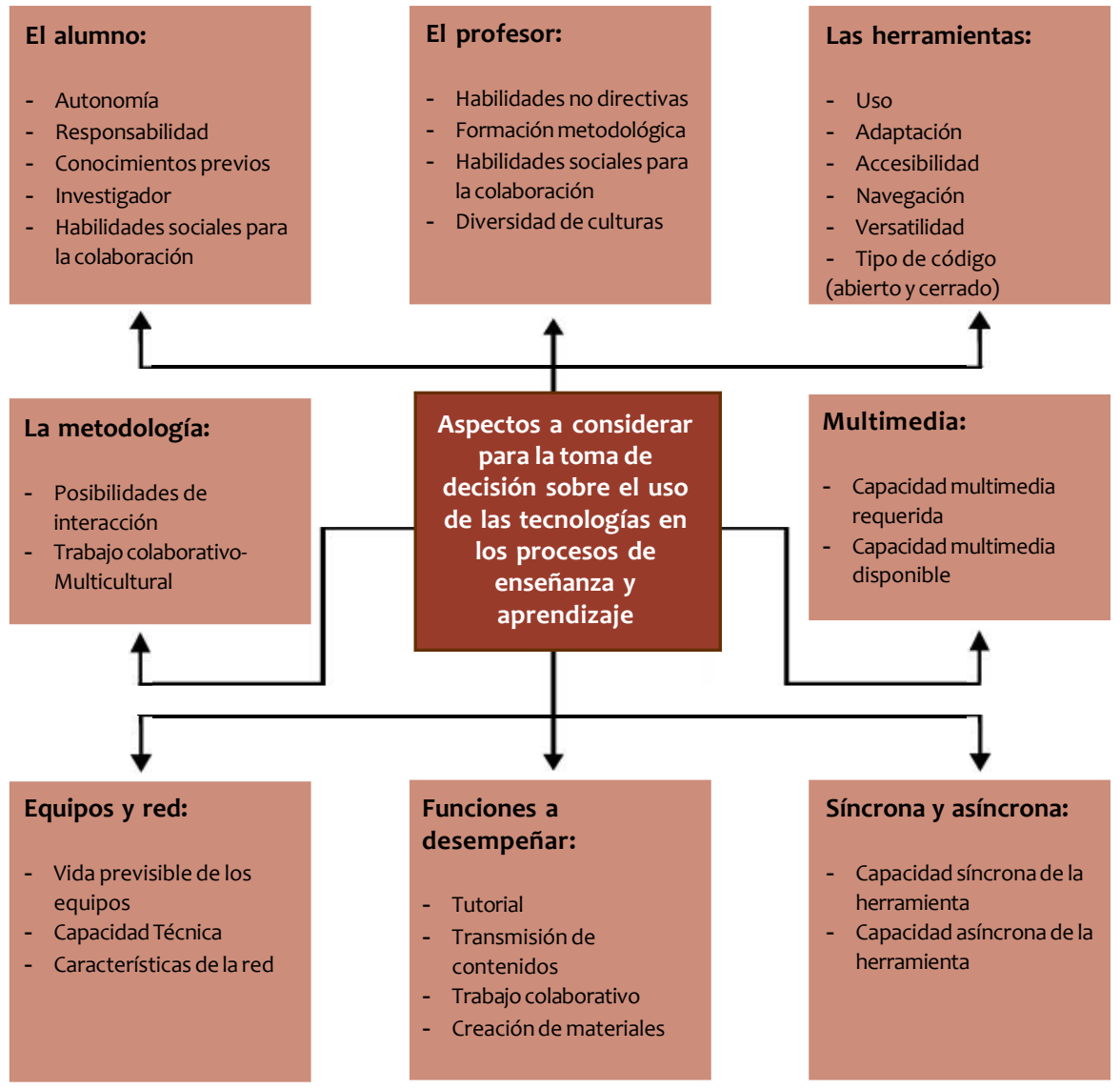

Figura 2- aspectos a considerar para la toma de decisiones sobre el uso de las tecnologías 
Debemos ver las tecnologías como un instrumento al servicio de la educación y que puede mejorar los desempeños, aumentar la motivación para aprender y alcanzar mejores resultados (Ramos, 2005).

La integración de las tecnologías en el proceso de enseñanza puede ser una excelente oportunidad para mejorar los sistemas educativos a partir de una buena planificación y preparación de nuevas situaciones, de lo contrario se pueden encontrar nuevos problemas de aprendizaje o reforzar los que ya existen (Cabero, 2006).

Todos los países desean mejorar la calidad y eficacia del aprendizaje escolar, apostar en las tecnologías como un medio para lograrlo (OCDE, $2001 \mathrm{~b}$ ).

En un informe sobre los desafíos de las tecnologías en la educación, la OCDE distingue tres series de argumentos para incluir las tecnologías en contexto educativo: económicos, sociales y pedagógicos.

En el argumento económico están elementos relacionados con la evolución de la economía mundial que valoriza la dimensión tecnológica, donde el conocimiento tecnológico, es siempre potenciador de fácil integración en el mercado de trabajo. Teniendo esta conciencia de la evolución de la economía mundial, los alumnos pueden tener un motivo adicional para el aprendizaje mediado por la tecnología.

Los argumentos sociales contemplan la capacidad de manejar las tecnologías como requisito esencial para participar en la sociedad revelándose fundamentalmente la lectura y el cálculo. En este argumento social se designa la alfabetización digital que abarca un gran abanico de competencias y procesos que las tecnologías fomentan y que se convierten en un requisito y derecho de los alumnos. 
Por fin, los argumentos pedagógicos se centran en el papel de las tecnologías en el proceso de enseñanza y aprendizaje. Las tecnologías pueden aumentar y enriquecer el aprendizaje gracias a la novedad y realismo que los actuales recursos presentan. La experiencia de la OCDE apunta el hecho de que los alumnos revelen desinterés por la escuela cuando ésta no les ofrece ambientes y experiencias tecnológicas.

Cruz (2009) apunta tres conjuntos de fundamentos para la integración curricular de las tecnologías:

- Desde el punto de vista del aprendizaje: considerando las principales teorías de aprendizaje que han venido a influenciar el uso educativo de las tecnologías en contexto educativo;

- Desde el punto de vista de la sociedad: considerando los datos que crean exigencias a nivel de la selección de contenidos adecuados a los interese de los ciudadanos;

- Desde el punto de vista de la investigación: dando configuración a los datos que nos permitan aclarar las perspectivas teóricas subyacentes al uso de las TIC al servicio del aprendizaje escolar, así como los beneficios derivados de dicho uso.

Ponte (2002) apunta como ventaja de integración de las tecnologías en la educación el hecho de permitir el acceso a la información, además permitir la producción de información, ser un excelente medio de comunicación a distancia , una herramienta para el trabajo colaborativo y promover formas de interacción social.

Para Paiva (2002), las tecnologías poseen un valor añadido para el proceso educativo permitiendo:

- Ganar tiempo en las tareas rutinarias;

- Posibilitan la formación a distancia, participación en trabajos y experiencias conjuntas a escala nacional e internacional; 
- Interactuar de forma diferencial entre profesor y alumno;

- Realizar investigación online dirigida ;

- Posibilidad de comunicación por email.

Un estudio sobre el impacto de las tecnologías en las escuelas europeas (Balanskat, Blamire \& Kefala, 2006) refiere que:

- Las tecnologías producen un impacto positivo en el desempeño escolar;

- Alumnos, profesores y padres de familia consideran que las tecnologías producen un impacto positivo sobre el aprendizaje de los alumnos;

- El uso de las tecnologías mejora los niveles de capacidades escolares en Inglés;

- Las escuelas que integren las tecnologías en su currículo de forma dinámica presentan mejores resultados que las escuelas que no lo hacen;

- Los mejores impactos (retornos) de las inversiones en tecnología ocurren en escuelas con ambientes más favorables a su integración (cultura escolar, tipos de liderazgo, etc.)

- Los profesores reconocen que los alumnos están más motivados y atentos cuando los recursos tecnológicos son usados en el aula de clases (Lemos, 2011);

- Los alumnos mejoran sus comportamientos y el desarrollo de competencias;

- Las tecnologías favorecen el aprendizaje diferenciado que permite ir al encuentro de las necesidades de cada alumno;

- Las tecnologías favorecen igualmente el aprendizaje independiente que es entendido como factor de autonomía y responsabilidad;

- Las tecnologías promueven el trabajo en equipo y la colaboración entre alumnos y entre profesores;

- El software de calidad que enriquezca el aprendizaje es también importante para el desarrollo. 
Por el conjunto de ventajas enumeradas, fácilmente entendemos su potencial motivador que puede imprimir a los aprendizajes. La ausencia de motivación de los alumnos es una realidad con la cual los profesores se enfrentan y que interfiere en la calidad de los aprendizajes. Sin embargo, las tecnologías pueden contribuir para mejorar los niveles de motivación y la concentración de los alumnos. Estudios de Lemos (2011) revelan que las tecnologías mejoran los niveles de concentración, de organización, del empeño, de la participación y del interés de los alumnos por el trabajo desarrollado en el aula de clases después de la introducción de las tecnologías.

La idea que, por ejemplo, las computadoras promueven el aislamiento social y reducen la creatividad, son dos mitos que Amante (2007) desmiente. Para la autora, las computadoras han demostrado ser promotoras de interacción y del trabajo corporativo creando oportunidades para el desarrollo de competencias sociales y cognitivas. Además, rechaza la idea de que el uso de las computadoras transforma a los niños en sujetos pasivos. La autora se refiere incluso que cuando se acusan a las computadoras de limitar la creatividad de los niños, lo primero que ocurre es cuestionar a la escuela, diciendo: antes que no existían las computadoras, era creativa. De cualquier modo, Amante (2007) no tiene dudas sobre el hecho que la computadora puede ser usada de manera no creativa.

Además de la contribución de las tecnologías en el área de las conductas, Amante (2007) y Ramos (2007) reconocen que las mismas estimulan el desarrollo del lenguaje, del pensamiento matemático y que pueden tener una gran contribución para la educación multicultural. Veamos algunos ejemplos:

- Al nivel del desarrollo del lenguaje:

- La interacción con las computadoras aumenta la comunicación verbal y la colaboración entre los niños;

- Las tecnologías proporcionan situaciones de conflicto socio-cognitivo propiciadoras de aprendizaje; 
- Al nivel del lenguaje escrito, el uso de procesadores de texto permite que los niños exploren y construyan conocimientos sobre representación simbólica;

- Los libros interactivos pueden tener una fuerte contribución para el desarrollo del vocabulario, de la sintaxis y del reconocimiento de palabras;

- El correo electrónico promueve la comunicación entre los alumnos y adultos a través de mensajes con imágenes, dibujos o historias.

- Al nivel del pensamiento matemático:

- Las tecnologías estimulan la emergencia de algunos conceptos como el reconocimiento de formas, recuento y clasificación;

- Desarrollo del pensamiento geométrico y espacial, favoreciendo el desarrollo de conceptos de simetría, patrones y organización espacial (Clements\&Swaminathan, 1995);

- Las tecnologías permiten que los alumnos produzcan objetos y actúen sobre ellos (aumentar o disminuir el tamaño, unir formas, colorear espacios)

Aun podemos aumentar dos tipos de ventajas de la integración de las tecnologías en la educación (Costa \& Jorge, 2011):

- Desarrollo de competencias relacionadas con la creatividad e innovación, con la autonomía y con el espíritu de iniciativa;

- Al nivel de la comunicación y colaboración, teniendo en cuenta la superación de barreras entre los diferentes intervinientes de la comunidad educativa, sea entre los propios docentes (aislamiento profesional), sea entre escuelas, o entre éstas y el resto de la comunidad educativa.

Un estudio de Rasinen (2003) que involucra la comparación de la forma como las tecnologías eran integradas en el currículo de seis países (Australia, Inglaterra, Francia, Países Bajos, Suecia y Estados 
Unidos) reveló que, por ejemplo en Australia, donde el currículo es basado en una lógica de que las personas enfrentan diariamente innúmeras situaciones con la interacción con la tecnología. De esa manera, las competencias adquiridas a lo largos del proceso educativo deben permitir que los alumnos:

- Desarrollen espíritu crítico y aplicación de nuevas ideas;

- Encuentren soluciones innovadoras para la comunidad;

- Logren conectarse con la incertidumbre;

- Puedan cooperar entre sí;

- Aprecien las diferencias culturales;

- Logren aprender a lo largo de la vida;

- Conozcan varias redes de información local, nacional e internacional.

En la secuencia de este estudio de Rasinen (2003), juzgamos que la gran ventaja de interacción de la tecnología en la educación es el desarrollo de múltiples competencias en los alumnos que les permitan ser mejores ciudadanos, no únicamente al nivel de la adquisición de conocimientos escolares sino, sobre todo, en su relación con el mundo.

En suma, y para que veamos, el enorme impacto que la tecnología puede tener, desde es integrada perfectamente en la educación, es el hecho del estudio del EuropeanSchoolnet(Balanskat, Blamire\&Kefala, 2006) revelar que en los países de la OSDE ${ }^{12}$ existe una asociación positiva entre el tiempo dedicado al uso educativo de la tecnología y el desempeño de los alumnos en pruebas de matemática del PISA ${ }^{13}$.

\footnotetext{
${ }^{12}$ Organización para la Cooperación y Desarrollo Económico, http://www.oecd.org/

13 Programme for International Student Assessment, http://www.oecd.org/pisa/
} 


\section{Referencias Bibliográficas}

AMANTE, L. (2007). Infância, escola e novas tecnologias. In Costa, F., Peralta, H. e Viseu, S. (Orgs.). As TIC na Educação em Portugal, Concepções e Práticas. Lisboa: Porto Editora.

BALANSKAT, A., BLAMIRE, R. \& KEFALA, S. (2006). The ICT Impact Report: a review studies of ICT impact on schools in Europe. European Schoolnet.

CABERO, J. (2006). Las nuevas tecnologias en la sociedad de la información. En J. Cabero (Coord.) Nuevas tecnologias aplicadas a la educación. Madrid: McGraw-Hill, 1-19.

COSTA, F. \& JORGE, M. (2011): Aprender e inovar com TIC em Portugal. Propostas e Desafios. Centro de Competência da Universidade do Minho.

LEMOS, M. (2011). A utilização das TIC em sala de aula : contributo para melhorar a motivação dos alunos. Dissertação de Mestrado. Universidade Católica Portuguesa.

MIRANDA, G. (2007). Limites e possibilidades das TIC na educação. Sísifo. Revista de Ciências da Educação, 3, pp. 41-50. Em http:// sisifo.fpce.ul.pt.

OCDE (2001a). Learning to Change: ICT in Schools. OECD, Paris 2001.

OCDE (2001b). Los desafios de las tecnologías de la información en la educación. Organización para la Cooperación y Desarrollo Económicos (OCDE) y Ministerio de Educación, Cultura y Deporte. España.

PAIVA, J. (2002). As tecnologias de informação e comunicação: utilização pelos professores. Departamento de Avaliação Prospectiva e Planeamento, Ministério da Educação de Portugal. PAPERT, S. (1987). Desafio a la mente. Computadoras y educación. 
Buenos Aires, Galápago.

RAMOS, J. (2005). Experiências educativas enriquecedoras no âmbito das tecnologias de informação e comunicação em Portugal: contributos para uma reflexão. In Silva, R. e Silva, A. (Orgs.). Educação, aprendizagem e tecnologia: um paradigma para o século XXI. Lisboa: Edições Sílabo.

RAMOS, J. (2007). Reflexões sobre a utilização educativa dos computadores e da Internet na escola. In Costa, F., Peralta, H. e Viseu, S. (Orgs.). As TIC na Educação em Portugal, Concepções e Práticas. Lisboa: Porto Editora.

RASINEN, A. (2003). An Analysis of the Technology Education Curriculum of Six Countries. Journal of Technology Education, Fall 2003 Volume 15, Number 1.

UNESCO (2004). Integrating ICT into Education. A Collective Case Study of Six Asian Countries. UNESCO Asia and Pacific Regional Bureau for Education. Bangkok, Thailand. 